\title{
A Pienkowsky-Type Calibration Scheme for 5211zl Weight Series Using Two Knife-Edge Direct-Reading Balances
}

\author{
H. S. Peiser
}

(July 15, 1964)

\begin{abstract}
To supplement information given in an earlier paper (J. Res. NBS 66C (Eng. and Instr.) No. 1, 33 (1962)) a Pienkowsky-type series is presented for the calibration of a set of weights having a combined mass of 10 units and individual masses of $5,2,1,1$, and $\Sigma 1$ where $\Sigma 1$ may represent a group of weights the sum of whose masses is one. The scheme includes the usual observational and computational checks, especially those against standard weights included in the series. Effects of linear balance drift are eliminated from the calibration of the unknown weights.
\end{abstract}

\section{Introduction}

Weight calibration schemes were discussed generally by Almer et al. ${ }^{1}$ After giving examples of Pienkowsky-type schemes the authors stated that such weighing series can be designed by a user to fit any combination of weights. In practice it has not been adequately easy for readers to design satisfactory Pienkowsky-type series. Fortunately the examples given in the earlier paper directly apply to most weight sets. The only important exception concerns weight sets made by German manufacturers

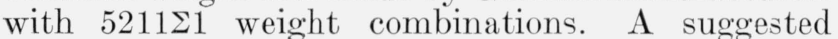
weighing sequence for $5211 \Sigma 1$ series is therefore now published using the nomenclature and definitions given by Almer et al.

1 H. E. Almer, L. B. Macurdy, H. S. Peiser, and E. A. Weck, Weight calibration schemes for two knife-edge direct-reading balances, J. Res. NBS 66C, (Eng. and Instr.) No. 1, 33 (1962).

\section{Weighing Scheme}

In addition to the (5), (2), (1) 1 , and $(1)_{2}$, and $\Sigma(1)$ weights, standard $\ldots 5$, and $\ldots 2$ weights are included as well as any 3 weight of good constancy. This 3 weight is not evaluated in the series and is here designated $\mathrm{C} 3$. The weighing sequence is given in table 1. $\Sigma(10)=(5)+(2)+(1)_{1}+(1)_{2}+\Sigma(1)$ is assumed to have been previously evaluated; lts correction is a constraint as previously discussed. The range in sums $(c+l),(d+h),(e+i),(f+j),(g+k)$, and $(l+m)$ provide a precision check; ideally all the sums should be identical. $(b+m)-(c+n)$ is another precision check; ideally this difference should be zero. The check on balance sensitivity is provided by the comparison of the difference $\left(n^{\prime}-n\right)$ with the accepted mass of the sensitivity weight.

The mass corrections of the weights to be determined are now given by the suggested solutions

TABLE 1

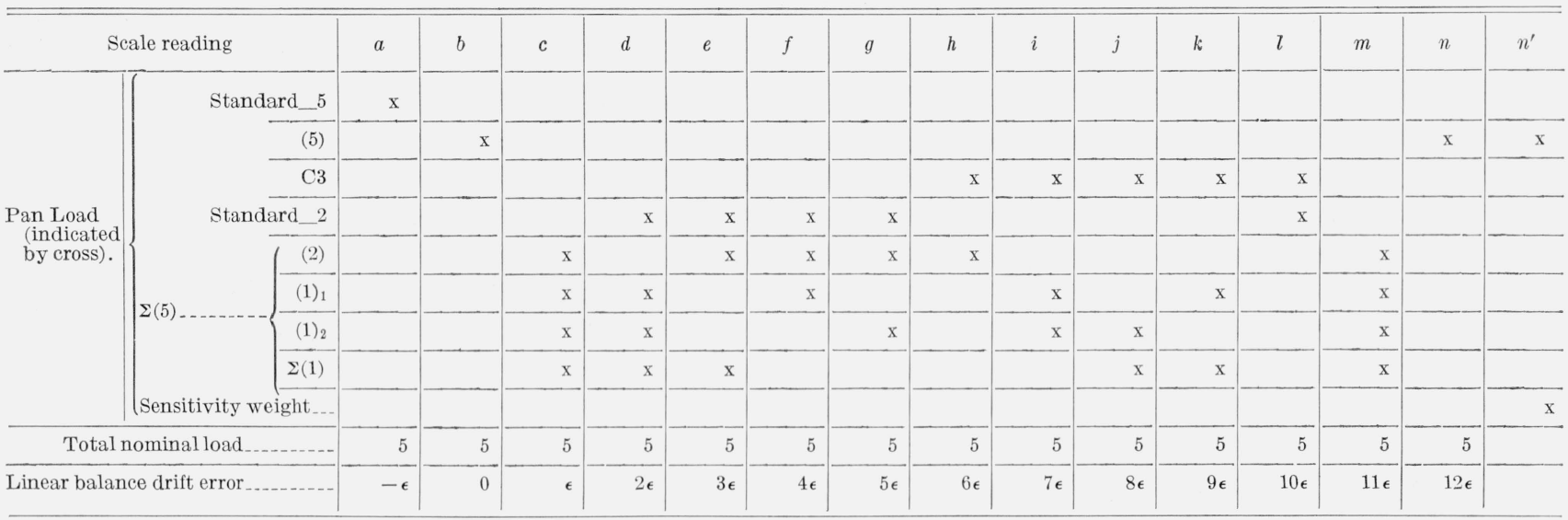




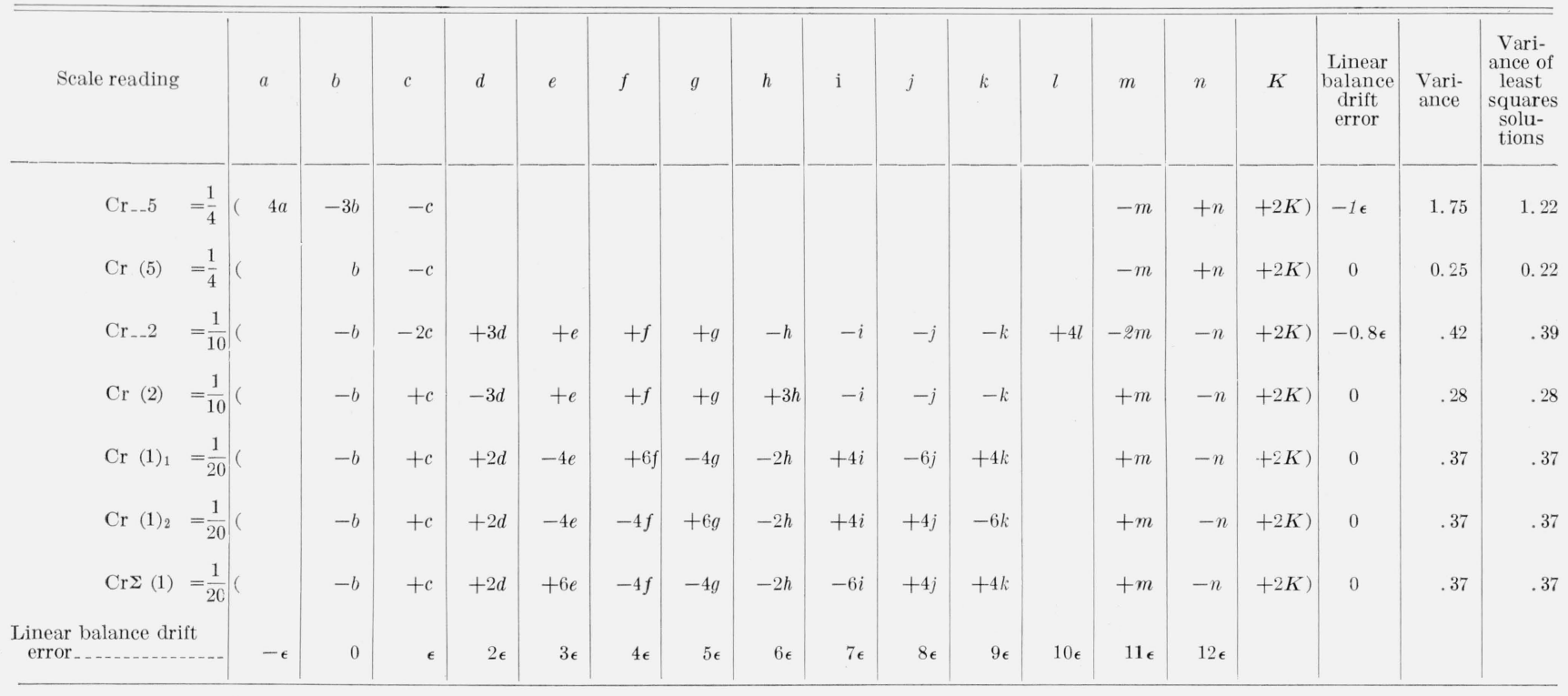

The sum of the coefficients for $a$ through $n$ must vanish for each line.

given in table 2. The solutions have conveniently small coefficients yet are very close to least squares solutions.

Optimum trend elimination for linear balance drift is given for all unknown weights but not for the standard weights, so that the values obtained for the standard weights are more likely to be affected by drift.

The correction values for the standards are compared with the accepted values.

Convenient computational checks are:

$$
\begin{array}{r}
\operatorname{Cr}(1)_{1}-\operatorname{Cr}(1)_{2}=\frac{1}{2}(f-g-j+k) \\
\operatorname{Cr} \Sigma(1)-\operatorname{Cr}(1)_{2}=\frac{1}{2}(e-g-i+k) \\
\operatorname{Cr}(2)-\operatorname{Cr}_{-} 2=\frac{1}{2}(c-d+h-l) \\
\operatorname{Cr}(1)+\operatorname{Cr} \Sigma(1)-\operatorname{Cr}_{-} 2=\frac{1}{2}(c-g+k-l) \\
\operatorname{Cr}(1)_{1}+\operatorname{Cr}(1)_{2}-\operatorname{Cr}(2)=\frac{1}{2}(d-e-h+i)
\end{array}
$$

The series has six degrees of freedom calculated as described in the previous paper.
It should be noticed that during the series all weights other than the standard $\ldots-5$ and C3 are put on the balance more than once which reduces the likelihood of false weight placings remaining undetected. Consistent with that requirement the series is designed to reduce interchanges of weights for successive observations.

Although the responsibility for this weighing scheme rests solely with the author, he is deeply conscious of having drawn freely on the superior knowledge and experience of other past and present members of the National Bureau of Standards: especially A. T. Pienkowsky, L. B. Macurdy, J. M. Cameron, and Mrs. M. E. Jones. 